\title{
PRINCIPAL BUNDLES OVER FINITE FIELDS
}

\author{
INDRANIL BISWAS AND S. SUBRAMANIAN
}

\begin{abstract}
Let $M$ be an irreducible smooth projective variety defined over $\overline{\mathbb{F}_{p}}$. Let $\varpi\left(M, x_{0}\right)$ be the fundamental group scheme of $M$ with respect to a base point $x_{0}$. Let $G$ be a connected semisimple linear algebraic group over $\overline{\mathbb{F}_{p}}$. Fix a parabolic subgroup $P \subsetneq$ $G$, and also fix a strictly anti-dominant character $\chi$ of $P$. Let $E_{G} \longrightarrow M$ be a principal $G$-bundle such that the associated line bundle $E_{G}(\chi) \longrightarrow E_{G} / P$ is numerically effective. We prove that $E_{G}$ is given by a homomorphism $\varpi\left(M, x_{0}\right) \longrightarrow G$. As a consequence, there is no principal $G$-bundle $E_{G} \longrightarrow M$ such that degree $\left(\varphi^{*} E_{G}(\chi)\right)>0$ for every pair $(Y, \varphi)$, where $Y$ is an irreducible smooth projective curve, and $\varphi: Y \longrightarrow E_{G} / P$ is a nonconstant morphism.
\end{abstract}

\section{INTRODUCTION}

We recall a question of $\mathrm{S}$. Keel: Let $X$ be a smooth projective surface over $\overline{\mathbb{F}_{p}}$ such that $L . C>0$ for every complete curve $C$. The question of Keel asks whether $L$ is ample. (See [Ke, p. 3959, Question 0.9].)

If the base field is complex numbers, then this question has a negative answer as shown by Mumford [Ha1, p. 56, Example 10.6]. Further examples were constructed in [Su1] and [MS] for higher dimensional complex projective varieties and also for varieties defined over fields of positive characteristics (these fields are not countable).

The varieties in all these examples are total spaces of flag bundles associated to vector bundles on curves, and the line bundles are the naturally associated ones. Proposition 1.2 shows that these type of varieties and line bundles never produce examples that would give a negative answer to the question of Keel.

Let $M$ be an irreducible smooth projective variety defined over $\overline{\mathbb{F}_{p}}$. Let $\varpi\left(M, x_{0}\right)$ be the fundamental group scheme with respect to a base point $x_{0} \in M$. The fundamental group scheme was introduced by Nori [No1], No2]. Let $G$ be a connected semisimple linear algebraic group over $\overline{\mathbb{F}_{p}}$. Fix a parabolic proper subgroup $P \subset G$, and also fix a strictly anti-dominant character $\chi$ of $P$. Given a principal $G$-bundle $E_{G} \longrightarrow M$, the quotient map $E_{G} \longrightarrow E_{G} / P$ defines a principal $P$-bundle. Let $E_{G}(\chi) \longrightarrow E_{G} / P$ be the line bundle associated to this principal $P$-bundle for the character $\chi$.

We prove the following theorem (see Theorem 4.1):

Theorem 1.1. Let $E_{G}$ be a principal $G$-bundle over $M$ such that the line bundle

$$
E_{G}(\chi) \longrightarrow E_{G} / P
$$

2000 Mathematics Subject Classification. 14L15, 14F05.

Key words and phrases. Finite fields, fundamental group scheme, ample line bundle, principal bundle. 
is numerically effective. Then $E_{G}$ is given by a homomorphism $\varpi\left(M, x_{0}\right) \longrightarrow G$.

The following proposition is proved using Theorem 1.1 (see Proposition 4.2):

Proposition 1.2. There is no principal $G$-bundle $E_{G}$ over $M$ such that the line bundle $E_{G}(\chi) \longrightarrow E_{G} / P$ has the following property: for every pair of the form $(Y, \varphi)$, where $Y$ is an irreducible smooth projective curve, and $\varphi: Y \longrightarrow E_{G} / P$ is a nonconstant morphism, the inequality

$$
\operatorname{degree}\left(\varphi^{*} E_{G}(\chi)\right)>0
$$

holds.

\section{Preliminaries}

Fix a prime $p$. Let $M$ be an irreducible smooth projective variety of dimension $d$ defined over $\overline{\mathbb{F}_{p}}$. Let

$$
F_{M}: M \longrightarrow M
$$

be the absolute Frobenius morphism of $M$. For any integer $n \geq 1$, let

$$
F_{M}^{n}:=\overbrace{F_{M} \circ \cdots \circ F_{M}}^{n \text {-times }}: M \longrightarrow M
$$

be the $n$-fold iteration of $F_{M}$; by $F_{M}^{0}$ we will denote the identity morphism of $M$.

For any $c \in \mathrm{CH}^{d}(M)$, let

$$
[c] \in \mathbb{Z}
$$

be the degree of $c(d=\operatorname{dim} M)$.

Fix a very ample line bundle $\mathcal{O}_{M}(1)$ on $M$. The degree of a torsionfree coherent sheaf $V$ on $M$ is defined to be

$$
\operatorname{degree}(V):=\left[c_{1}(V) \cdot c_{1}\left(\mathcal{O}_{M}(1)\right)^{d-1}\right] \in \mathbb{Z} .
$$

We note that

$$
\operatorname{degree}(V)=\operatorname{degree}\left(\left.V\right|_{D}\right),
$$

where $D$ is a smooth complete intersection curve obtained by intersecting $(d-1)$ hyperplanes on $M$ from the complete linear system $\left|\mathcal{O}_{M}(1)\right|$. Let $U \subset M$ be a Zariski open subset such that the codimension of the complement $M \backslash U$ is at least two. For a torsionfree coherent sheaf $E \longrightarrow U$, define

$$
\operatorname{degree}(E)=\operatorname{degree}\left(\iota_{*} E\right),
$$

where $\iota: U \hookrightarrow M$ is the inclusion map; note that $\iota_{*} E$ is a coherent sheaf.

A Zariski open subset $U \subset M$ such that the codimension of the complement $M \backslash U$ is at least two will be called a big open subset.

Let $G$ be a connected semisimple linear algebraic group defined over $\overline{\mathbb{F}_{p}}$. Let $E_{G}$ be a principal $G$-bundle over $M$. Let $(Q, U, \sigma)$ be a triple, where

- $Q \subset G$ is a maximal proper parabolic subgroup,

- $U \subset M$ is a big open subset, and 
- $\sigma: U \longrightarrow E_{G} / Q$ is a reduction of the structure group, over $U$, to the subgroup $Q$.

The principal $G$-bundle $E_{G}$ is called semistable if for all such triples, the inequality

$$
\operatorname{degree}\left(\sigma^{*} T_{\text {rel }}\right) \geq 0
$$

holds, where $T_{\text {rel }} \longrightarrow E_{G} / Q$ is the relative tangent bundle for the projection $E_{G} / Q \longrightarrow$ $M$. (See [Ra], [RR], [RS].)

A principal $G$-bundle $E_{G} \longrightarrow M$ is called strongly semistable if the principal $G$-bundle $\left(F_{M}^{n}\right)^{*} E_{G}$ is semistable for all $n \geq 0$.

We recall that a character $\chi$ of a parabolic subgroup $P \subset G$ is called strictly antidominant if the associated line bundle

$$
G(\chi):=G \times^{P} \overline{\mathbb{F}_{p}} \longrightarrow G / P
$$

is ample.

\section{RESTRICTION OF PRINCIPAL BUNDLES TO CURVES}

We start with a simple lemma.

Lemma 3.1. Let $E_{G} \longrightarrow M$ be a principal $G$-bundle such that for every pair of the form $(Y, \varphi)$, where $Y$ is an irreducible smooth projective curve, and $\varphi: Y \longrightarrow M$ is a morphism, the principal $G$-bundle $\varphi^{*} E_{G} \longrightarrow Y$ is semistable. Then $\varphi^{*} E_{G}$ and $E_{G}$ are strongly semistable.

Proof. Let $F_{C}$ be the absolute Frobenius morphism of $C$. Replacing $\varphi$ by $\varphi \circ F_{C}^{n}$ in the given condition, we conclude that $\left(\varphi \circ F_{C}^{n}\right)^{*} E=\left(F_{C}^{n}\right)^{*}\left(\varphi^{*} E\right)$ is semistable. Hence $\varphi^{*} E$ is strongly semistable. Now from (2.1) it follows that $E$ is strongly semistable.

Proposition 3.2. Let $E_{G} \longrightarrow M$ be a principal $G$-bundle such that for every pair of the form $(C, \varphi)$, where $C$ is an irreducible smooth projective curve, and

$$
\varphi: C \longrightarrow M
$$

is a morphism, the principal $G$-bundle $\varphi^{*} E_{G} \longrightarrow C$ is semistable. Then

$$
\left[c_{2}\left(\operatorname{ad}\left(E_{G}\right)\right) \cdot c_{1}\left(\mathcal{O}_{M}(1)\right)^{d-2}\right]=0 .
$$

Proof. We will first prove this for vector bundles. Let $E \longrightarrow M$ be a vector bundle such that the pulled back vector bundle $\varphi^{*} E \longrightarrow C$ is semistable for every pair $(C, \varphi)$ of the above type.

From Lemma 3.1 we know that $\varphi^{*} E \longrightarrow C$ is strongly semistable. Hence the endomorphism bundle $\operatorname{End}\left(\varphi^{*} E\right)=\varphi^{*} \operatorname{End}(E)$ is semistable [RR, p. 288, Theorem 3.23]. From this it follows that $\operatorname{End}(E)$ is numerically effective. To prove this, take any morphism

$$
\gamma: C \longrightarrow \mathbb{P}(\operatorname{End}(E))
$$


and set $\varphi=h \circ \gamma$, where $h: \mathbb{P}(\operatorname{End}(E)) \longrightarrow M$ is the natural projection. Note that $\gamma^{*} \mathcal{O}_{\mathbb{P}(\operatorname{End}(E))}(1)$ is a quotient of $\varphi^{*} \operatorname{End}(E)$. Since $\varphi^{*} E n d(E)$ is semistable of degree zero, we have

$$
\operatorname{degree}\left(\gamma^{*} \mathcal{O}_{\mathbb{P}(\operatorname{End}(E))}(1)\right) \geq 0,
$$

proving that $\operatorname{End}(E)$ is numerically effective.

If $M$ is a curve then there is nothing to prove. Take a smooth complete intersection surface

$$
\iota: S \hookrightarrow M
$$

obtained by intersecting $(d-2)$ hyperplanes on $M$ from the complete linear system $\left|\mathcal{O}_{M}(1)\right|$. If $M$ is a surface, then take $S=M$. Let

$$
W:=\iota^{*} \operatorname{End}(E)
$$

be the restriction of $E n d(E)$ to $S$. We note that

$$
\left[c_{2}(\operatorname{End}(E)) \cdot c_{1}\left(\mathcal{O}_{M}(1)\right)^{d-2}\right]=\left[c_{2}(W)\right] \in \mathbb{Z} .
$$

Also, note that $c_{1}(W)=0$ because $W=W^{*}$.

Substituting $W$ for $E$ in Lemma 3.1 we conclude that $W$ is strongly semistable. Therefore, we have the Bogomolov inequality for $W$

$$
\left[c_{2}(W)\right] \geq 0
$$

(see [Bo], [La]); recall that $c_{1}(W)=0$.

Let

$$
f: Z:=\mathbb{P}\left(\iota^{*} \operatorname{End}(E)\right) \longrightarrow S
$$

be the projective bundle parametrizing the hyperplanes in the fibers of $\iota^{*} E n d(E)=W$. The tautological line bundle $\mathcal{O}_{Z}(1)$ over $Z$ will be denoted by $L$.

The Grothendieck's construction of Chern classes gives

$$
\left[f^{*} c_{2}(W) \cdot c_{1}(L)^{r-2}\right]-\left[f^{*} c_{1}(W) \cdot c_{1}(L)^{r-1}\right]+\left[c_{1}(L)^{r}\right]=0
$$

(see [Ha2, page 429]), where $r=\operatorname{rank}(W)$, and $f$ is the projection in (3.4) to the surface $S$. Therefore,

$$
\left[f^{*} c_{2}(W) \cdot c_{1}(L)^{r-2}\right]+\left[c_{1}(L)^{r}\right]=0
$$

because $c_{1}(W)=0$.

The line bundle $L \longrightarrow \mathbb{P}(W)$ is numerically effective because it is a restriction of the numerically effective line bundle $\mathcal{O}_{\mathbb{P}(\operatorname{End}(E))}(1)$. Consequently, $\left[c_{1}(L)^{r}\right] \geq 0$.

Since $\left[c_{1}(L)^{r}\right] \geq 0$, from (3.5) we conclude that $\left[c_{2}(W)\right] \leq 0$. Comparing this with (3.3) we conclude that $\left[c_{2}(W)\right]=0$. Hence from (3.2) we conclude that

$$
\left[c_{2}(\operatorname{End}(E)) \cdot c_{1}\left(\mathcal{O}_{M}(1)\right)^{d-2}\right]=0 .
$$

Now we consider the general case of principal $G$-bundles. Let $E_{G}$ be as in the statement of the proposition. 
Let $m$ be the dimension of $G$. Let $\operatorname{ad}\left(E_{G}\right)$ be the adjoint vector bundle. The line bundle $\bigwedge^{m} \operatorname{ad}\left(E_{G}\right)$ is trivial because the adjoint action of $G$ on $\bigwedge^{m} \operatorname{Lie}(G)$ is trivial. In particular, $c_{1}\left(\operatorname{ad}\left(E_{G}\right)\right)=0$, and hence

$$
c_{2}\left(\operatorname{End}\left(\operatorname{ad}\left(E_{G}\right)\right)\right)=2 m \cdot c_{2}\left(\operatorname{ad}\left(E_{G}\right)\right) .
$$

Since $\varphi^{*} E_{G}$ is strongly semistable (Lemma 3.1), the adjoint vector bundle $\operatorname{ad}\left(\varphi^{*} E_{G}\right)=$ $\varphi^{*} \operatorname{ad}\left(E_{G}\right)$ is semistable [RR, p. 288, Theorem 3.23]. Hence from (3.6) we know that

$$
\left[c_{2}\left(\operatorname{End}\left(\operatorname{ad}\left(E_{G}\right)\right)\right) \cdot c_{1}\left(\mathcal{O}_{M}(1)\right)^{d-2}\right]=0 .
$$

Therefore, (3.7) implies that $\left[c_{2}\left(\operatorname{ad}\left(E_{G}\right)\right) \cdot c_{1}\left(\mathcal{O}_{M}(1)\right)^{d-2}\right]=0$. This completes the proof of the proposition.

\section{Fundamental GRoup SCHEME AND PRINCIPAL BUNDLES}

Fix a base point $x_{0} \in M$. Let $\varpi\left(M, x_{0}\right)$ be the fundamental group scheme [No1, No2]. There is a universal principal $\varpi\left(M, x_{0}\right)$-bundle

$$
F_{\varpi\left(M, x_{0}\right)} \longrightarrow M
$$

Given a homomorphism $\rho: \varpi\left(M, x_{0}\right) \longrightarrow G$, we get a principal $G$-bundle $F_{\varpi\left(M, x_{0}\right)}(G)$ over $M$ by extending the structure group of $F_{\varpi\left(M, x_{0}\right)}$ using $\rho$.

Fix a parabolic subgroup $P \subsetneq G$. Fix a strictly anti-dominant character $\chi$ of $P$.

Given a principal $G$-bundle $E_{G} \longrightarrow M$, the quotient map $E_{G} \longrightarrow E_{G} / P$ defines a principal $P$-bundle. Let $E_{G}(\chi) \longrightarrow E_{G} / P$ be the line bundle associated to this principal $P$-bundle for the character $\chi$.

Theorem 4.1. Let $E_{G}$ be a principal $G$-bundle over $M$ such that the line bundle $E_{G}(\chi)$ over $E_{G} / P$ is numerically effective. Then $E_{G}$ is given by a homomorphism $\varpi\left(M, x_{0}\right) \longrightarrow$ $G$.

Proof. Take any pair $(C, \theta)$, where $C$ is an irreducible smooth projective curve, and

$$
\theta: C \longrightarrow M
$$

is a morphism. Consider the fiber bundle

$$
E_{G} / P \longrightarrow M
$$

Note that $\theta^{*}\left(E_{G} / P\right)=\left(\theta^{*} E_{G}\right) / P$, and the pullback of the line bundle $E_{G}(\chi)$ to $\theta^{*}\left(E_{G} / P\right)$ coincides with the line bundle $\left(\theta^{*} E_{G}\right)(\chi)$ associated to the principal $P$-bundle $\theta^{*} E_{G} \longrightarrow$ $\left(\theta^{*} E_{G}\right) / P$ for the character $\chi$. Since $E_{G}(\chi)$ is numerically effective, and the pullback of a numerically effective line bundle is numerically effective, we conclude that the line bundle

$$
\left(\theta^{*} E_{G}\right)(\chi) \longrightarrow\left(\theta^{*} E_{G}\right) / P
$$

is numerically effective. This implies that the principal $G$-bundle $\theta^{*} E_{G}$ is semistable $[\mathrm{BP}$, p. 766, Theorem 3.1].

Therefore, from Lemma 3.1 and Proposition 3.2 we conclude that $E_{G}$ is strongly semistable, and

$$
\left[c_{2}\left(\operatorname{ad}\left(E_{G}\right)\right) \cdot c_{1}\left(\mathcal{O}_{M}(1)\right)^{d-2}\right]=0
$$


Hence $E_{G}$ is a given by a homomorphism $\varpi\left(M, x_{0}\right) \longrightarrow G$ [Bi, pp. 210-211, Theorem 1.1] (for vector bundles this was proved earlier in [Su2]). This completes the proof of the theorem.

Proposition 4.2. There is no principal $G$-bundle $E_{G}$ over $M$ such that the line bundle $E_{G}(\chi) \longrightarrow E_{G} / P$ has the following property: for every pair of the form $(Y, \varphi)$, where $Y$ is an irreducible smooth projective curve, and $\varphi: Y \longrightarrow E_{G} / P$ is a nonconstant morphism, the inequality degree $\left(\varphi^{*} E_{G}(\chi)\right)>0$ holds.

Proof. Let $E_{G} \longrightarrow M$ be a principal $G$-bundle such that

$$
\operatorname{degree}\left(\varphi^{*} E_{G}(\chi)\right)>0
$$

for every pair $(Y, \varphi)$ of the above type. From Theorem 4.1 we know that $E_{G}$ is given by a homomorphism

$$
\rho: \varpi\left(M, x_{0}\right) \longrightarrow G
$$

Fix a faithful representation

$$
\eta: G \hookrightarrow \operatorname{GL}\left(V_{0}\right) .
$$

Let $E_{G}\left(V_{0}\right):=E_{G} \times{ }^{G} V_{0} \longrightarrow M$ be the associated vector bundle. Since $E_{G}$ is given by the homomorphism $\rho$ in (4.2), the vector bundle $E_{G}\left(V_{0}\right)$ is given by the homomorphism

$$
\eta \circ \rho: \varpi\left(M, x_{0}\right) \longrightarrow \mathrm{GL}\left(V_{0}\right) .
$$

In particular, $E_{G}\left(V_{0}\right)$ is an essentially finite vector bundle [No1], [No2]. Therefore, there is a finite morphism

$$
f: \widetilde{M} \longrightarrow M
$$

where $\widetilde{M}$ is an irreducible smooth projective variety of dimension $d$, such that the pulled back vector bundle $f^{*} E_{G}\left(V_{0}\right)$ is trivial [BH, p. 557].

Since $f^{*} E_{G}\left(V_{0}\right)$ is trivial, the principal GL $\left(V_{0}\right)$-bundle $\left(f^{*} E_{G}\right) \times^{G} \operatorname{GL}\left(V_{0}\right) \longrightarrow \widetilde{M}$, which is the extension of structure group of $f^{*} E_{G}$ by $\eta$, is trivial. So the reduction of structure group

$$
f^{*} E_{G} \hookrightarrow\left(f^{*} E_{G}\right) \times^{G} \mathrm{GL}\left(V_{0}\right)=\widetilde{M} \times \mathrm{GL}\left(V_{0}\right)
$$

is given by a morphism $\widetilde{M} \longrightarrow \mathrm{GL}\left(V_{0}\right) / G$. Since $G$ is semisimple, the quotient $\mathrm{GL}\left(V_{0}\right) / G$ is an affine variety, hence there is no nonconstant morphism to it from $\widetilde{M}$. Therefore, the principal $G$-bundle $f^{*} E_{G}$ is trivializable. Fix a trivialization of it.

Fixing a point $z_{0}$ of $G / P$, we construct the constant section passing through $z_{0}$

$$
\sigma: \widetilde{M} \longrightarrow\left(f^{*} E_{G}\right) / P=\widetilde{M} \times(G / P)
$$

of the projection $\left(f^{*} E_{G}\right) / P \longrightarrow \widetilde{M}$. The composition $\beta \circ \sigma: \widetilde{M} \longrightarrow E_{G} / P$, where

$$
\beta:\left(f^{*} E_{G}\right) / P=f^{*}\left(E_{G} / P\right) \longrightarrow E_{G} / P
$$

is the natural map, has the property that the line bundle

$$
(\beta \circ \sigma)^{*} E_{G}(\chi) \longrightarrow \widetilde{M}
$$


is trivial. Indeed, $\beta^{*} E_{G}(\chi)$ is the pullback of the line bundle $G(\chi)$ (defined in (2.3)) to $\left(f^{*} E_{G}\right) / P=\widetilde{M} \times(G / P)$ by the natural projection. Since $(\beta \circ \sigma)^{*} E_{G}(\chi)$ is trivial, the assumption that $E_{G}(\chi)$ satisfies the condition in (4.1) is contradicted. This completes the proof of the proposition.

\section{REFERENCES}

[Bi] I. Biswas: On principal bundles over a projective variety defined over a finite field, Jour. K-Theory 4 (2009), 209-221.

[BH] I. Biswas and Y. I. Holla: Comparison of fundamental group schemes of a projective variety and an ample hypersurface, Jour. Alg. Geom. 16 (2007), 547-597.

[BP] I. Biswas and A. J. Parameswaran: A criterion for the strongly semistable principal bundles over a curve in positive characteristic, Bull. Sci. math. 128 (2004), 761-773.

[Bo] F. A. Bogomolov: Holomorphic tensors and vector bundles on projective varieties, Math. USSR Izv. 13 (1979), 499-555.

[Ha1] R. Hartshorne: Ample subvarieties of algebraic varieties, Lect. Notes in Math. 156, SpringerVerlag, Berlin-Heidelberg-New York, 1970.

[Ha2] R. Hartshorne: Algebraic Geometry, Graduate Texts in Mathematics, No. 52, Springer-Verlag, New York, 1977.

[Ke] S. Keel: Polarized pushouts over finite fields, Comm. Alg. 31 (2003), 3955-3982.

[La] A. Langer: Semistable sheaves in positive characteristic, Ann. of Math. 159 (2004), 251-276.

[MS] V. Mehta and S. Subramanian: Nef line bundles which are not ample, Math. Zeit. 219 (1995), $235-244$.

[No1] M. V. Nori: On the representations of the fundamental group scheme, Compos. Math. 33 (1976), $29-41$

[No2] M. V. Nori: The fundamental group-scheme, Proc. Ind. Acad. Sci. (Math. Sci.) 91 (1982), 73-122.

[RR] S. Ramanan and A. Ramanathan: Some remarks on the instability flag, Tôhoku Math. Jour. 36 (1984), 269-291.

[Ra] A. Ramanathan: Stable principal bundles on a compact Riemann surface, Math. Ann. 213 (1975), $129-152$.

[RS] A. Ramanathan and S. Subramanian: Einstein-Hermitian connections on principal bundles and stability, Jour. Reine Angew. Math. 390 (1988), 21-31.

[Su1] S. Subramanian: Mumford's example and a general construction, Proc. Ind. Acad. Sci. (Math. Sci.) 99 (1989), 197-208.

[Su2] S. Subramanian: Strongly semistable bundles on a curve over a finite field, Arch. Math. 89 (2007), $68-72$.

School of Mathematics, Tata Institute of Fundamental Research, Homi Bhabha Road, BOMBAY 400005, INDIA

E-mail address: indranil@math.tifr.res.in

School of Mathematics, Tata Institute of Fundamental Research, Homi Bhabha Road, BOMBAY 400005, INDIA

E-mail address: subramnn@math.tifr.res.in 